\title{
Bounds on Direct Couplings of Superheavy Metastable Particles to the Inflaton Field from Ultra High Energy Cosmic Ray Events
}

\author{
${ }^{a}$ Instituto de Física, DFN - USP \\ Rua do Matão, Trav. R, 187 \\ 05508-900 São Paulo, SP, Brazil \\ ${ }^{b}$ Instituto de Física Teórica - UNESP \\ Rua Pamplona, 145 \\ 01405-900 São Paulo, SP, Brazil \\ ${ }^{c}$ Departamento de Raios Cósmicos e Cronologia \\ Universidade Estadual de Campinas - Unicamp \\ CP 6165, 13083-970, Campinas - SP, Brazil
}

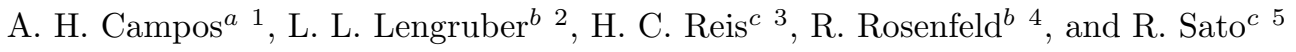

\begin{abstract}
Top-down models for the origin of ultra high energy cosmic rays (UHECR's) propose that these events are the decay products of relic superheavy metastable particles, usually called $X$ particles. These particles can be produced in the reheating period following the inflationary epoch of the early Universe. We obtain constraints on some parameters such as the lifetime and direct couplings of the $X$-particle to the inflaton field from the requirement that they are responsible for the observed UHECR flux.
\end{abstract}

PACS Categories: 98.70.Sa, 98.80.Cq

\footnotetext{
${ }^{1}$ hcampos@charme.if.usp.br

${ }^{2}$ leticia@ift.unesp.br

${ }^{3}$ hreis@ifi.unicamp.br

${ }^{4}$ rosenfel@ift.unesp.br

${ }^{5}$ rsato@ifi.unicamp.br
} 


\section{Introduction}

The ultra high energy cosmic rays (UHECR's), events with primary energies above $E>10^{20} \mathrm{eV}$, constitute one of the most intriguing mysteries in nature. Their origin, source and composition are not understood since they were first observed by the Volcano Ranch experiment in 1963 [1].

According to conventional theories, cosmic rays are produced by the acceleration of charged particles in intense electromagnetic fields of astrophysical objects. This conventional wisdom, however, has some difficulties in explaining the observed flux of UHECR's. Most of the known astrophysical objects do not have an electromagnetic field strong enough neither a large acceleration site capable to accelerate particles up to $10^{19} \mathrm{eV}$ [2]-四.

Another problem is the propagation of the UHECR's through the intergalactic medium. If the particles are protons or nuclei, they should quickly degrade their energy due to interactions with the cosmic microwave background (CMB), producing a cut in the spectrum at energies above $3 \times 10^{9} \mathrm{eV}$, the so-called GZK limit [5]-[7]. Simulations [8] show that in order that protons with energy larger than $10^{20} \mathrm{eV}$ arrive at Earth they must have traveled less than $\approx 100 \mathrm{Mpc}$, the attenuation length. A cut off is also expected if the primary particles are high energy photons in the range of $3 \times 10^{12} \mathrm{eV}$ to $10^{22} \mathrm{eV}$, which have attenuation length smaller than $100 \mathrm{Mpc}$, due to their interaction with various background photons (infrared/optical, CMB or universal radio background), mainly by pair production and inverse Compton scattering. These consecutive interactions produce electromagnetic cascades with decreasing energy that end up below 100 $\mathrm{GeV}$ resulting in a gamma-ray background.

Furthermore, UHECR's are usually not deflected by intergalactic magnetic fields, so their incoming directions should point to the sources. However, taking into account the arrival directions of the UHECR events [9] and constraining their origin to be in a distance of $100 \mathrm{Mpc}$ around the Earth, no candidate sources were found so far (an exception is a suggestion that these events can be generated in M87 galaxy in the Virgo Cluster and made isotropic by the galactic magnetic field $[10$ ).

A new scenario, the so-called top-down mechanism, was then proposed to explain these events 11. In this scenario, UHECR's would originate from the decay of some superheavy particles $\left(m_{X} \gg 10^{20} \mathrm{eV}\right)$ inside the GZK volume. They could be either emitted by topological defects at the present epoch [12] or created in the pos-inflationary era [13]. In the second case, where the supermassive particles must be metastable $\left(\tau_{X} \geq 10^{10}\right.$ years $)$, they could have been created perturbatively, by a mechanism that is called reheating [14], or by noperturbative process like preheating [15], instant preheating [16] or gravitational production [17]. In this letter we will study bounds on the direct coupling of $X$ particles produced during the reheating phase to the inflaton field by requiring that they are sources of UHECR's. A preliminary version of this work appeared in 18$]$. 


\section{Observed UHECR's flux and $X$-particle abun- dance}

We will now derive a relation among the abundance $\Omega_{X}$, the lifetime $\tau_{X}$ and the mass $m_{X}$ of the $X$-particle in order to provide the observed flux of UHECR's on Earth.

It is reasonable to assume that $X$-particles decay into quarks and leptons. Particles are produced following the quark-hadron fragmentation process described by QCD that results in the production of nucleons $(\approx 10 \%)$ and pions $(\approx 90 \%)$ distributed equally among the three charge states. Charged pions produce basically electrons and neutrinos and neutral pions decay into photons. Photons dominate the primary spectrum over protons by a factor of $\approx 6$ [19 - 20]. Following Sigl and Bhattacharjee [11], we will consider that the UHECR's are photons with energy $E \simeq 10^{20} \mathrm{eV}$ and an attenuation length of $l\left(E_{\gamma}\right) \approx 10 \mathrm{Mpc}$. It was recently suggested that photons can indeed provide the bulk of UHECR's without violating EGRET bounds on GeV gamma-ray backgrounds 21].

The photon flux generated by the decay of an uniform distribution of $X$ particles is given by:

$$
J_{\gamma}=\frac{1}{4 \pi} l\left(E_{\gamma}\right) \dot{n}_{X} \frac{d N_{\gamma}}{d E_{\gamma}}
$$

where $d N_{\gamma} / d E_{\gamma}$ is the photon injection spectrum from $X$-decay and $\dot{n}_{X}(=$ $\left.n_{X} / \tau_{X}\right)$ is the $X$-decay rate.

From the observed UHECR's flux [1], $J\left(E \simeq 10^{20} \mathrm{eV}\right)=10^{-27}\left(\mathrm{~m}^{2} \mathrm{sr} \mathrm{s} \mathrm{GeV}\right)^{-1}$ and using $d N_{\gamma} / d E_{\gamma} \approx E_{\gamma}^{-1.5}$ from a QCD model [11], one gets:

$$
\dot{n}_{X}=3.7 \times 10^{-46}\left(\frac{m_{X}}{10^{12} \mathrm{GeV}}\right)^{-1 / 2} \mathrm{~cm}^{-3} \mathrm{~s}^{-1} .
$$

So, we can obtain a relation between $\Omega_{X} h^{2}$ and $\tau_{X}$ :

$$
\tau_{X}=\frac{\Omega_{X} \rho_{c}}{\dot{n}_{X} m_{X}} \simeq 10^{22}\left(\Omega_{X} h^{2}\right)\left(\frac{10^{12} \mathrm{GeV}}{m_{X}}\right)^{1 / 2} \text { years },
$$

where $\Omega_{X}=\rho_{X} / \rho_{c}, \rho_{X}$ is the $X$-particle mass density, $\rho_{c} \simeq 10^{-5} h^{2} \mathrm{GeV} \mathrm{cm}^{-3}$ is the critical density, and $h$ is the present value of Hubble's constant in units of $100 \mathrm{~km} \mathrm{~s}^{-1} \mathrm{Mpc}^{-1}$.

It could be interesting that these unknown $X$-particles constitute a fraction of the cold dark matter (CDM). In this case, they could be concentrated in galactic haloes and should not be uniformly distributed along the whole universe, as assumed in (11). Considering just the contribution of our galactic halo the correct equation to describe the flux of UHECR's is:

$$
J_{\gamma}^{h}=\frac{1}{4 \pi} R^{h} \dot{n}_{X}^{h} \frac{d N_{\gamma}}{d E_{\gamma}},
$$


where $R^{h}$ is the radius of the galactic halo and $\dot{n}_{X}^{h}$ is the decay rate of the $X$-particles clustered in the halo.

The flux above can be written in terms of $J_{\gamma}$, given in (1), so that $J_{\gamma}^{h}=f J_{\gamma}$ and:

$$
\begin{aligned}
f & =\frac{J_{\gamma}^{h}}{J_{\gamma}}=\frac{n_{X}^{h} R^{h}}{n_{X} l\left(E_{\gamma}\right)}= \\
& =1.5 \times 10^{3}\left(\frac{0.2}{\Omega_{C D M} h^{2}}\right)\left(\frac{R^{h}}{100 \mathrm{Kpc}}\right)\left(\frac{10 \mathrm{Mpc}}{l\left(E_{\gamma}\right)}\right)\left(\frac{\rho_{C D M}^{h}}{0.3 \mathrm{GeV} \mathrm{cm}^{-3}}\right)
\end{aligned}
$$

where $\rho_{C D M}^{h}$ is the cold dark matter energy density in the halo. We recall that if $X$-particles are a fraction of CDM, it can be written as $\rho_{X}^{h}=\varepsilon \rho_{C D M}^{h}$. The fraction $\varepsilon$ is the same everywhere in the universe, so that $\varepsilon=\Omega_{X} / \Omega_{C D M}$.

The halo concentration of $X$-particles implies a modification of equation (3):

$$
\tau_{X}=10^{22} f \Omega_{X} h^{2}\left(\frac{10^{12} \mathrm{GeV}}{m_{X}}\right)^{1 / 2} \text { years }
$$

Considering $m_{X}=10^{12} \mathrm{GeV}$, for a minimum $\tau_{X}$ of $10^{10}$ years and a maximum $\Omega_{X} h^{2}$ of the order of 1 , we obtain the following limits (for $f \approx 10^{3}$ ) (see also [20]:

$$
\begin{array}{ll}
\text { for }\left(\Omega_{X} h^{2}\right) \simeq 1 & \rightarrow \tau_{X} \simeq 10^{25} \text { years, } \\
\text { for } \tau_{X} \simeq 10^{10} \text { years } & \rightarrow \quad\left(\Omega_{X} h^{2}\right) \simeq 10^{-15} .
\end{array}
$$

Therefore, cosmologically interesting abundances of X-particles which at the same time provide a solution to the UHECR events require a very large life-time $\tau_{X}$. These requirements are generally difficult to obtain in realistic theories, but examples do exist [22].

\section{Production of $X$-particles in the reheating phase}

In the inflationary scenario, following the exponential expansion of the universe, particles are created by the oscillation of inflaton field $\phi$ about its minimum. The coupling of the inflaton with other fields becomes important and allows the transference of its energy to other particles. In the perturbative reheating process [14], the inflaton decays mostly into relativistic particles (radiation) that reheat the universe. In this context, the superheavy particle $X$ could be created by either radiation annihilation process (indirect production) or by direct inflaton decay $\phi \rightarrow X \bar{X}$ (direct production), if such a coupling is allowed. The study of the latter mechanism is the main focus of this work. 
In order to study quantitatively $X$-particle production during reheating, we have to solve the coupled differential Boltzmann equations for the energy densities of $X$ particles $\left(\rho_{X}\right)$, radiation $\left(\rho_{R}\right)$ and inflaton $\left(\rho_{\phi}\right)$ :

$$
\begin{aligned}
& \dot{\rho_{\phi}}+3 H \rho_{\phi}+\Gamma_{\phi}=0, \\
& \dot{\rho_{R}}+4 H \rho_{R}-\left(1-B_{X}\right) \Gamma_{\phi} \rho_{\phi}-\frac{\langle\sigma|v|\rangle}{m_{X}}\left[\left(\rho_{X}\right)^{2}-\left(\rho_{X}^{e q}\right)^{2}\right]=0, \\
& \dot{\rho}_{X}+3 H \rho_{X}-\frac{m_{X}}{m_{\phi}} B_{X} \Gamma_{\phi} \rho_{\phi}+\frac{\langle\sigma|v|\rangle}{m_{X}}\left[\left(\rho_{X}\right)^{2}-\left(\rho_{X}^{e q}\right)^{2}\right]=0,
\end{aligned}
$$

where:

$$
H^{2}=\frac{8 \pi}{m_{p l}^{2}}\left(\rho_{\phi}+\rho_{R}+\rho_{X}\right) .
$$

In the above equations $H$ is the expansion rate of the universe, $B_{X}$ is the branching ratio for the $\phi \rightarrow X \bar{X}$ decay, $\rho_{X}^{e q}$ is the equilibrium value for the $X$ particle energy density and $\langle\sigma|v|\rangle$ is the thermal average of the $X$ annihilation cross section times the Møller flux factor. The third term in equation (9) has a factor $m_{X} / m_{\phi}$ that corrects a similar equation in [23]. According to [24], the annihilation cross section can be estimated as $\langle\sigma|v|\rangle \sim \alpha^{2} / T^{2}$ where $g=\sqrt{4 \pi \alpha}$ is the gauge coupling strength and $T$ is the characteristic temperature for the process. $\Gamma_{\phi}$ is the inflaton total width and is obtained as a function of the reheating temperature, $T_{R H}$, assuming instantaneous conversion of the inflaton energy density into radiation:

$$
\Gamma_{\phi}=1.93 \times 10^{-18}\left(\frac{T_{R H}}{\mathrm{GeV}}\right)^{2} \mathrm{GeV} .
$$

It is important to notice that the reheating temperature $\left(T_{R H}\right)$ is not the highest temperature achieved by the universe during reheating. For example, in this paper we are using a simple chaotic inflation model where $V=m_{\phi}^{2} \phi^{2} / 2$ and $m_{\phi}=10^{13} \mathrm{GeV}$. Following [23] it can be shown that, even if $T_{R H}=10^{9} \mathrm{GeV}$, the universe can achieve temperatures as high as $10^{12} \mathrm{GeV}$. In this case, for $g=1$ we have $\langle\sigma|v|\rangle=10^{-26} \mathrm{GeV}^{-2}$, which allows the process $\gamma \gamma \rightarrow X \bar{X}$.

It is convenient to work with co-moving and dimensionless variables and therefore we defined appropriate parameters [23] and solved the equations numerically for the scaled variable $X \equiv \rho_{X} m_{X}{ }^{-1} a^{3}$, where $a(t)$ is the scale factor. In this case, the new independent variable is $x=a m_{\phi}$.

In Figure 1, we illustrate the behavior of $X(x)$. The curves $\mathrm{A}, \mathrm{B}$ and $\mathrm{C}$ show two plateaus associated with two mechanisms of X-particle production: the first one is the indirect production $(\gamma \gamma \rightarrow X \bar{X})$ and the second is the direct one $(\phi \rightarrow X \bar{X})$. Modifying $B_{X}$ and keeping $T_{R H}$ constant (B and $\mathrm{C}$ curves) only the second plateau changes, reflecting the dependence of the direct decay 
on $B_{X}$. On the other hand, when $T_{R H}$ is much smaller than $10^{9} \mathrm{GeV}$, there is not enough energy for the indirect process and hence, the first plateau tends to disappear.

\section{Finding $\Omega_{X}$}

We can find the $X$-particle mass density $\Omega_{X}$ today using the present value $\left(x=x_{0}\right)$ of the radiation abundance $\Omega_{R}\left(\Omega_{R} h^{2}=4.3 \times 10^{-5}\right)$ :

$$
\frac{\Omega_{X} h^{2}}{\Omega_{R} h^{2}}=\frac{\rho_{X}\left(x_{0}\right)}{\rho_{R}\left(x_{0}\right)}
$$

In thermal equilibrium, $T \propto g_{*}{ }^{-1 / 3} a^{-1}$ [24], and hence $\rho_{R}$ can be written as:

$$
\rho_{R}\left(x_{0}\right)=\rho_{R}\left(x_{R H}\right)\left(\frac{g_{*}\left(x_{R H}\right)}{g_{*}(x)}\right)^{1 / 3}\left(\frac{x_{R H}}{x_{0}}\right)^{4}
$$

where $g_{*}$ is the effective number of degrees of freedom (we used $g_{*}\left(x_{R H}\right)=200$ ).

Assuming that $X$ particles are created already decoupled, we can write that $\rho_{X}\left(x_{0}\right)=\rho_{X}\left(x_{R H}\right)\left(x_{R H} / x_{0}\right)^{3}$, where:

$$
\rho_{X}\left(x_{R H}\right)=X_{F} x_{R H}{ }^{-3} m_{\phi} m_{X} .
$$

The parameter $X_{F}$ is the $X$ value in the second plateau (Figure 1) and corresponds to $X\left(x_{R H}\right)$. So, we get:

$$
\begin{aligned}
\Omega_{X} h^{2} & =\Omega_{R} h^{2} \frac{\rho_{X}\left(x_{R H}\right)}{\rho_{R}\left(x_{R H}\right)} \frac{T_{R H}}{T_{0}} \\
& =1.5 \times 10^{9}\left(\frac{T_{R H}}{\mathrm{GeV}}\right) \frac{X_{F} m_{X} m_{\phi}^{3}}{x_{i}^{3} H_{i}^{2} m_{p l}^{2}},
\end{aligned}
$$

where $m_{p l}$ is the Planck mass and $T_{0}=2.37 \times 10^{-13} \mathrm{GeV}$ is the present-epoch CMB temperature.

\section{Numerical Results}

We worked with acceptable reheating temperatures in the range of $10^{-2} \mathrm{GeV} \leq$ $T_{R H} \geq 10^{9} \mathrm{GeV}$. The lower limit is imposed by nucleosyntesis and the upper one is chosen not to lead to overproduction of gravitinos 25]. The subscript $i$ refers to the epoch before inflaton decay. For chaotic inflation models we have $H_{i} \approx m_{\phi}$. As initial condition for the differential equations we choose $x_{i}=1$.

Solving the Boltzmann equations numerically for different $B_{X}$ and $T_{R H}$, we obtain the respective $X_{F}$ and consequently its associated $\Omega_{X} h^{2}$, as it is shown in the table 1. 


\begin{tabular}{|c||c|c||c|c||c|c|}
\hline \multirow{2}{*}{\multicolumn{1}{|c||}{$B_{X}$}} & \multicolumn{2}{c||}{$T_{R H}=10^{9} \mathrm{GeV}$} & \multicolumn{2}{c||}{$T_{R H}=10^{3} \mathrm{GeV}$} & \multicolumn{2}{c|}{$T_{R H}=10^{-2} \mathrm{GeV}$} \\
\cline { 2 - 6 } & $X_{F}$ & $\Omega_{X} h^{2}$ & $X_{F}$ & $\Omega_{X} h^{2}$ & $X_{F}$ & $\Omega_{X} h^{2}$ \\
\hline 0 & $3.3 \times 10^{-6}$ & 0.38 & 0 & 0 & 0 & 0 \\
\hline $1 \times 10^{-15}$ & $1.8 \times 10^{-4}$ & 20.97 & $1.78 \times 10^{-4}$ & $2.06 \times 10^{-5}$ & $1.78 \times 10^{-4}$ & $2.06 \times 10^{-10}$ \\
\hline $1 \times 10^{-11}$ & 1.78 & $2.06 \times 10^{5}$ & 1.78 & $2.06 \times 10^{-1}$ & 1.78 & $2.06 \times 10^{-6}$ \\
\hline $1 \times 10^{-9}$ & $1.78 \times 10^{2}$ & $2.06 \times 10^{7}$ & $1.78 \times 10^{2}$ & $2.06 \times 10^{1}$ & $1.78 \times 10^{2}$ & $2.06 \times 10^{-4}$ \\
\hline $1 \times 10^{-5}$ & $1.78 \times 10^{6}$ & $2.06 \times 10^{11}$ & $1.78 \times 10^{6}$ & $2.06 \times 10^{5}$ & $1.78 \times 10^{6}$ & 2.06 \\
\hline $1 \times 10^{-3}$ & $1.78 \times 10^{8}$ & $2.06 \times 10^{13}$ & $1.78 \times 10^{8}$ & $2.06 \times 10^{7}$ & $1.78 \times 10^{8}$ & $2.06 \times 10^{2}$ \\
\hline
\end{tabular}

Table 1: Numerical results for the final abundance obtained by solving the Boltzmann equations, for $T_{R H}=10^{9}, 10^{3}, 10^{-2} \mathrm{GeV}$ and $B_{X}<10^{-3}$.

For $B_{X}=0$, the $X$ particle production is not effective if the reheating temperature is lower than $T=10^{9} \mathrm{GeV}$. From table 1 we also get the expressions below, related to the direct production process that prevails over the indirect one, for $B_{X}>10^{-16}$ :

$$
\begin{aligned}
X_{F} & =1.78 \times 10^{11} B_{X} \\
\Omega_{X} h^{2} & \simeq 2.06 \times 10^{7}\left(\frac{T_{R H}}{\mathrm{GeV}}\right) B_{X} .
\end{aligned}
$$

An excellent analytical approximation to the numerical results can be obtained by requiring that $n_{X}=n_{\phi} B_{X}$, in which case we find:

$$
X_{F}=\frac{3}{8 \pi} \frac{m_{p l}^{2}}{m_{\Phi}^{2}} B_{X}
$$

Substituting the numerical values the above equation reproduces (17) exactly.

The maximum efficiency of the direct decay process $(\phi \rightarrow X \bar{X})$ is obtained when $T_{R H}$ is minimum. Recently, low temperature reheating models were explored in [26]. Taking for the minimum value of $T_{R H} \simeq 10^{-2} \mathrm{GeV}$ and using the upper limit for $X$ abundance $\left(\Omega_{X} h^{2} \simeq 1\right)$ we get the maximum value for the branching ratio of $B_{X}=10^{-5}$.

More generally, we can find bounds on $B_{X}$ by requiring that $X$-particles are sources of UHECR's, in which case the limits of (6) apply. These bounds are shown in figure 2 as a function of the reheating temperature for two limits on the abundance: $\Omega_{X} h^{2} \leq 1\left(\tau_{X} \simeq 10^{25}\right.$ years $)$ and $\Omega_{X} h^{2} \geq 10^{-15}\left(\tau_{X} \simeq 10^{10}\right.$ years).

\section{Conclusion}

We have investigated the constraints on the properties of supermassive relic metastable particles demanding that their decay products are responsible for the observed UHECR flux. In particular, we found the relevant range for the 
branching ratio of inflaton decay into the $X$-particle as a function of the reheating temperature.

For very small branching ratio of the inflaton to the $X$-particle, $B_{X} \geq 10^{-16}$, the direct production $(\phi \rightarrow X \bar{X})$ dominates over the indirect thermal production even for $T_{R H}=10^{9} \mathrm{GeV}$. For the smallest possible reheat temperature, $T_{R H}=10^{-2} \mathrm{GeV}$, thermal production of $X$-particle does not occur and the direct mechanism with $B_{X} \leq 10^{-5}$ is required.

Further consequences of the top-down scenario are the dominance of photons and neutrinos as primaries of UHECR's 19 and an anisotropy of these events as a result of the large concentration of $X$-particles in the galactic center 27. More recently, it was suggested that clumps of dark matter in the halo could explain the observed multiplets (12 doublets and 2 triplets) of UHECR's events 28. A detailed analysis including several models for galactic haloes of our and other galaxies was performed in [29]. We look forward to new experimental results from current and future experiments to elucidate the composition and anisotropy of the UHECR events. In particular, the first site of the Auger Observatory in the southern hemisphere, being sensitive to the galactic center, will be important in testing the anisotropy resulting from the top-down scenario.

\section{Acknowledgments}

This work was supported by Fundação de Amparo à Pesquisa do Estado de São Paulo (FAPESP) and Conselho Nacional de Desenvolvimento Científico e Tecnológico (CNPq).

\section{References}

[1] J. Linsley, Phys. Rev. Lett. 10 (1963) 146.

[2] M. A. Lawrence et al., J. Phys. G Nucl. Part. Phys. 17 (1991) 733.

[3] N. Hayashida et al., Phys. Rev. Lett. 73 (1994) 3491.

[4] D. J. Bird et al., Phys. Rev. Lett. 71 (1993) 3401.

[5] K.Greisen, Phys. Rev. Lett. 16 (1966) 748.

[6] G. T. Zatsepin and V. A. Kuzmin, JETP Lett. 4 (1966) 78.

[7] P. H. Frampton, e-print astro-ph/9804219.

[8] F. A. Aharonian and J. W. Cronin, Phys. Rev. D50 (1994) 1892.

[9] E. Waxmann and J. Miralda-Escudé, Astrophys. J. Lett. L89 (1996) 472. 
[10] E-J. Ahn, G. Medina-Tanco, P. L. Biermann and T. Stanev, Nucl. Phys. Proc. Suppl. 87, (2000) $41 \%$.

[11] For a review, see P. Bhattacharjee and G. Sigl, Phys. Rept. 327 (2000) 109.

[12] C. T. Hill, D. N. Schramm and T. P. Walker, Phys. Rev. D36 (1987) $100 \%$.

[13] V. A. Kuzmin and V. A. Rubakov, Phys. Atom. Nucl. 61 (1998) 1028; V. Berezinsky, M. Kachelrieß and A. Vilenkin, Phys. Rev. Lett. 79 (1997) 4302.

[14] A. D. Dolgov and A. D. Linde, Phys. Lett. 116B, (1983) 329; L. F. Abbott, E. Fahri and M. B. Wise, Phys. Lett. 117B, (1982) 29.

[15] A. Dolgov and D. Kirilova, Sov. Nucl. Phys. 51, (1990) 273; J. Traschen and R. Brandenberger, Phys. Rev. D42 (1990) 2491; L. Kofman, A. D. Linde and A. A. Starobinsky, Phys. Lett. 73, (1994) 3195.

[16] G. Felder, L. Kofman and A. Linde, Phys. Rev. D59 (1999) 123523.

[17] L. H. Ford, Phys. Rev. D35 (1987) 2955.

[18] L. L. Lengruber and R. Rosenfeld, Proceedings of the 11th International Symposium on Very High Energy Cosmic Ray Interactions, Campinas, Brazil, July 2000, Nucl. Phys. Proc. Suppl. 97 (2001) 278.

[19] V. Berezinsky, P. Blasi and A. Vilenkin, Phys. Rev. D58 (1998) 103515.

[20] M. Birkel and S. Sarkar, Astropart. Phys. 9 (1998) $29 \%$.

[21] O. E. Kalashev, V. A. Kuzmin, D. V. Semikoz and I. I. Tkachev, e-print astro-ph/0107130.

[22] See, e.g., P. H. Frampton, B. Keszthelyi and Y. J. Ng, Int. J. Mod. Phys. D8 (1999) 11\%; K. Benakli, J. Ellis and D. V. Nanopolous, Phys. Rev. D59 (1999) 047301; Y. Uheara, e-print hep-ph/0107297.

[23] D. J. H. Chung, E. W. Kolb, A. Riotto, Phys. Rev. D60 (1999) 63504.

[24] E. W. Kolb and M. Turner, The Early Universe. Addison-Wesley, 1990.

[25] L. M. Krauss, Nucl. Phys. B227, (1983) 556; J. Ellis, J. Kim and D. V. Nanopoulos, Phys. Lett. 145B, (1984) 181; M. Yu. Khlopov and A. D. Linde, Phys. Lett. 138B, (1984) 265.

[26] G. F. Giudice, E. W. Kolb, A. Riotto, Phys. Rev. D64, (2001) 023508.

[27] G. A. Medina-Tanco and A. A. Watson, Astropart. Phys. 12, (1999) 25; V. Berezinsky and A. Mikhailov, Phys. Lett. B449, (1999) 23\%;

[28] P. Blasi and R. K. Sheth, Phys. Lett. B486, (2000) 233.

[29] W. Evans, F. Ferrer and S. Sarkar, e-print astro-ph/0103085. 


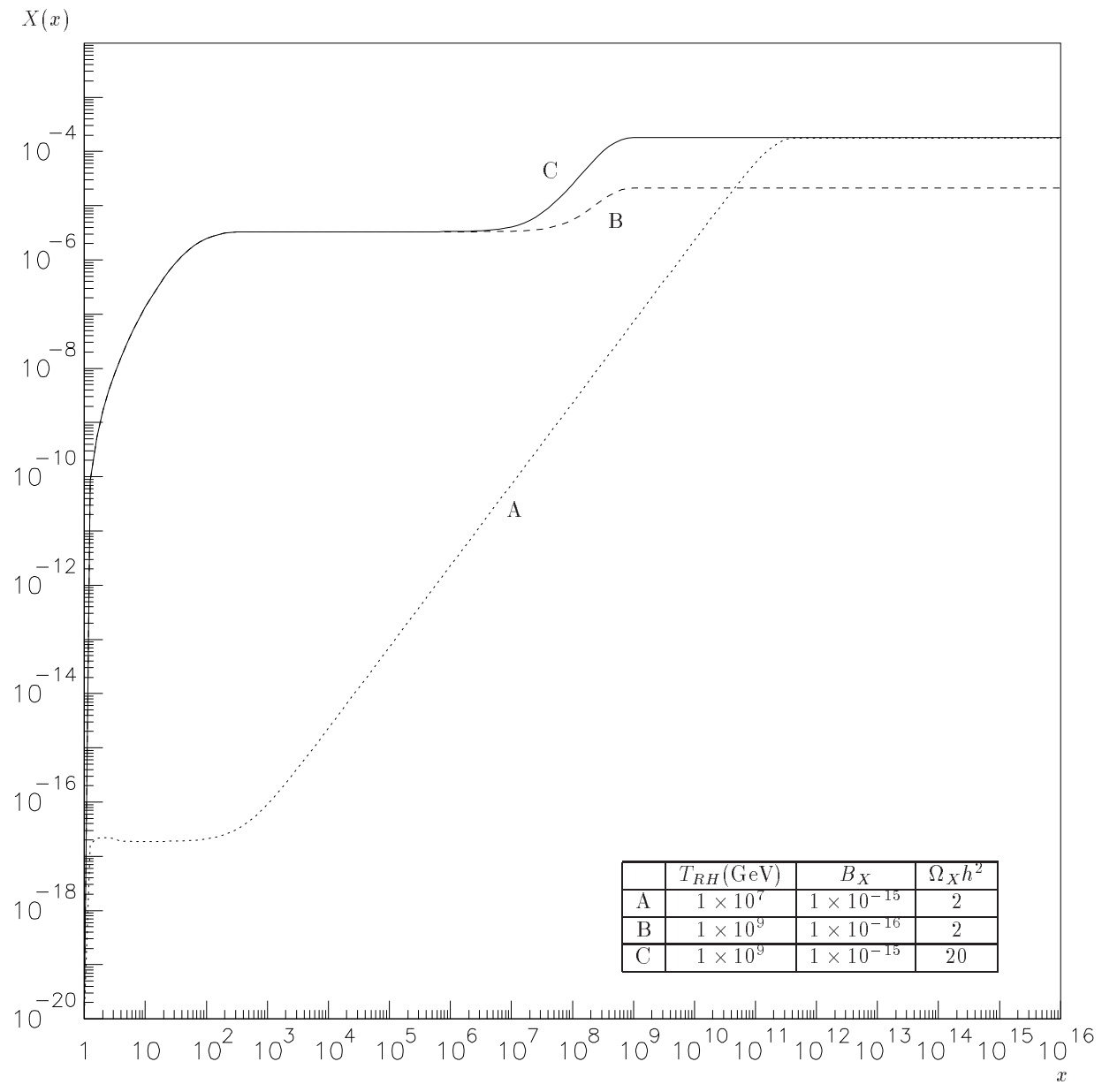

Figure 1: Evolution of $X\left(\equiv \rho_{X} m_{X}^{-1} a^{3}\right)$ as a function of the modified scale factor, $x$. We used $m_{\phi}=10^{13} \mathrm{GeV}$ and $m_{X}=10^{12} \mathrm{GeV}$. 


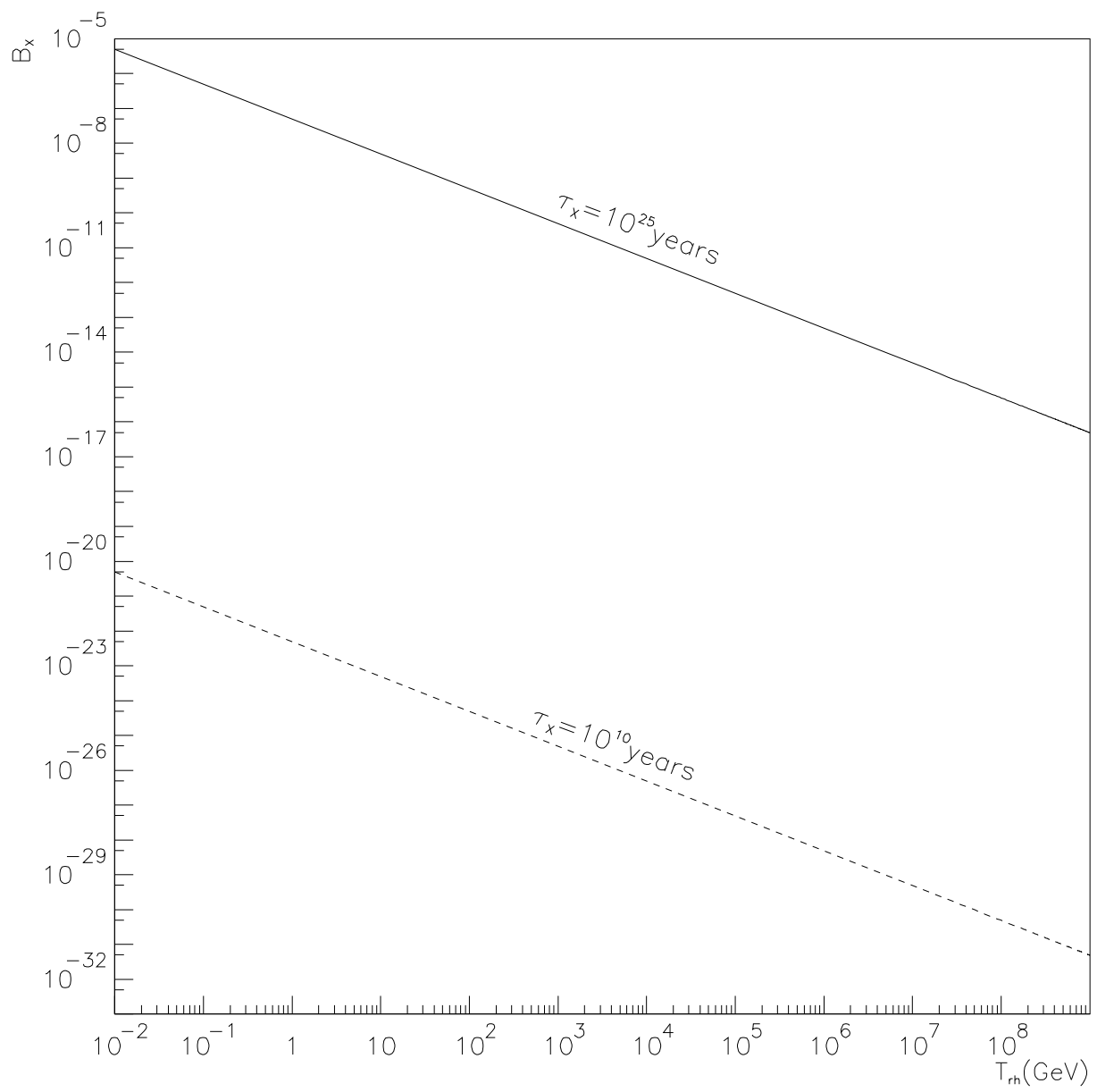

Figure 2: Branching ratio $B_{X}$ of the inflaton into $X$-particles as a function of the reheating temperature $T_{R H}$ for two limiting values of $\Omega_{X} h^{2}$. 